\title{
Dermoscopy of Generalized Eruptive Histiocytosis: Case Report and Brief Review of the Literature
}

\author{
Danijela Dobrosavljevic, ${ }^{1,2}$ Jovana Majstorovic, ${ }^{1}$ Martina Bosic ${ }^{2,3}$
}

\author{
1 Clinic of Dermatovenereology, Clinical Centre of Serbia, Belgrade, Serbia \\ 2 Faculty of Medicine, University of Belgrade, Serbia \\ 3 Institute of Pathology, University of Belgrade, Serbia
}

Key words: generalized eruptive histiocytosis, non-Langerhans cell histiocytosis, histiocytic disorders, dermoscopy, dermatoscopy Citation: Dobrosavljevic D, Majstorovic J, Bosic M. Dermoscopy of generalized eruptive histiocytosis: case report and brief review of the literature. Dermatol Pract Concept. 2020;10(3):e2020057. DOI: https://doi.org/10.5826/dpc.1003a57

Accepted: February 27, 2020; Published: June 29, 2020

Copyright: $\odot 2020$ Dobrosavljevic et al. This is an open-access article distributed under the terms of the Creative Commons Attribution License, which permits unrestricted use, distribution, and reproduction in any medium, provided the original author and source are credited.

Funding: None.

Competing interests: The authors have no conflicts of interest to disclose.

Authorship: All authors have contributed significantly to this publication.

Corresponding author: Danijela Dobrosavljevic, MD, PhD, Clinic of Dermatovenereology, Pasterova 2 St, Clinical Centre of Serbia and Faculty of Medicine, University of Belgrade, Belgrade, Serbia. Email: danijela_dobrosavljevic@yahoo.co.uk

\section{Introduction}

Generalized eruptive histiocytosis (histiocytoma) (GEH) is a very rare cutaneous non-Langerhans cell histiocytosis, characterized by recurrent crops of small red to brown papules.

\section{Case Presentation}

A 58-year-old woman with an 8-month history of hundreds of symmetric yellow-brown flat-topped papules (Figure 1, $\mathrm{A}$ and $\mathrm{B}$ ) came for dermatologist consultation. The lesions appeared in crops localized on the trunk and extremities. It was believed that the skin lesions were caused by trazodone and lithium, which were being used in the treatment of bipolar disorder. The medications were stopped, but the lesions continued to develop. Routine blood and urine analyses were unremarkable.

Dermoscopy was performed on one representative lesion on the lower leg. The histology was consistent with the diagnosis of GEH (Figure 1, C-G).
After GEH diagnostics, additional investigations were done. Hyperprolactinemia, hypercorticism, and hypofunction of the thyroid gland were detected. Abdominal ultrasonography and MRI of the sella turcica and hematological studies with biopsy of the bone marrow were without pathological findings. Three years from the first onset, the skin lesions mostly resolved, leaving hyperpigmented macules.

\section{Literature Review}

Seventy-four cases $(58.1 \%$ males) of GEH have been published, including $24(32.4 \%)$ children (Table 1 and Supplementary References, which are appended to the pdf). The average age was 30.5 years. In adults and children the average age was 43.2 years and 4.2 years, respectively. The most frequent body site was the trunk $(86 \%)$, followed by the extremities (79\%). The lesions resolved spontaneously from 2 weeks onward, but in rare cases persisted for 20 years. In 4 children the lesions evolved into xanthoma disseminatum; in 1 child the lesions coexisted with juvenile xanthogranuloma. 

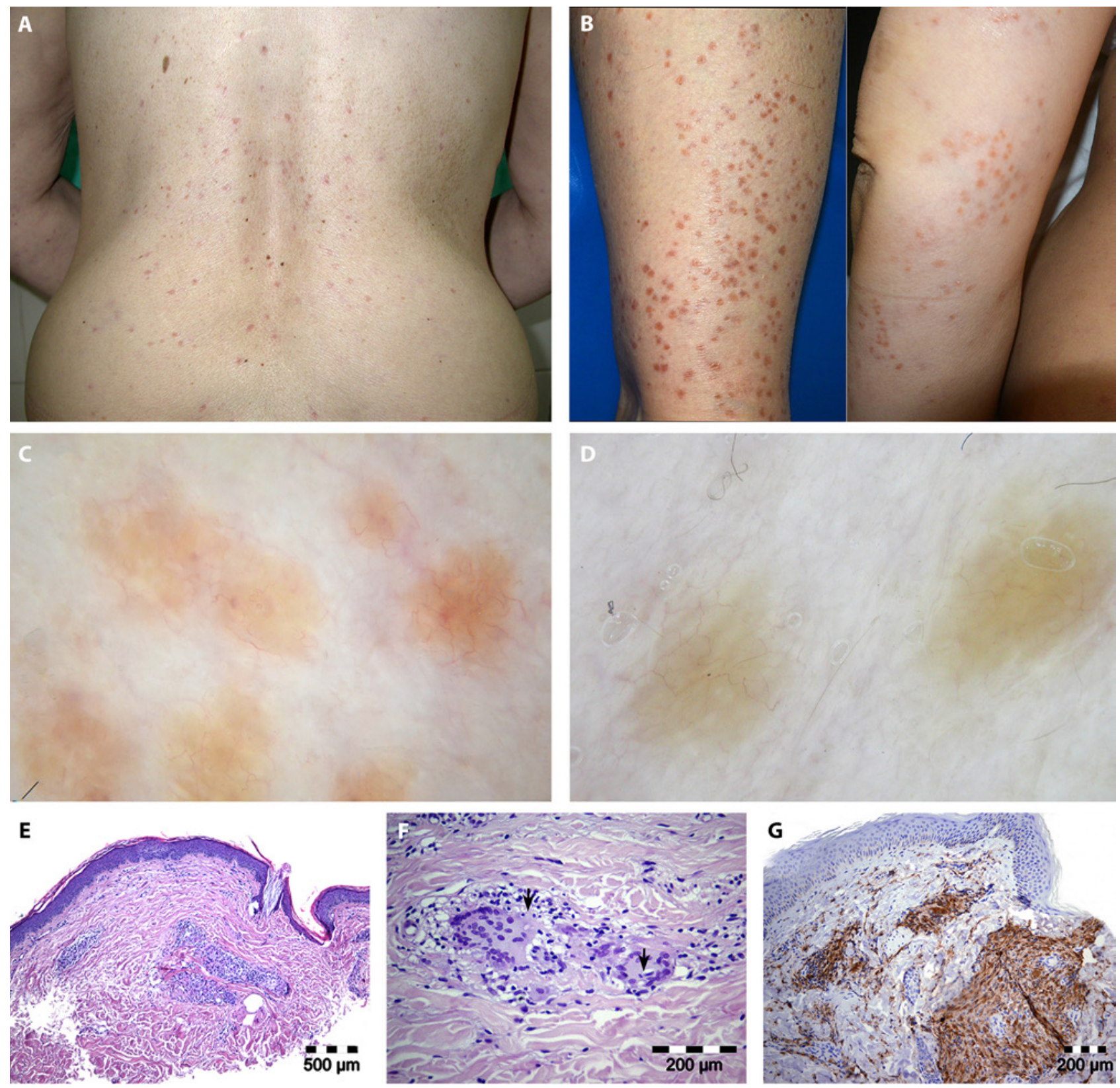

Figure 1. (A,B) Symmetric yellow-brown, flat-topped papules localized on the trunk (A) and extremities (B). (C) Dermoscopic finding: orange-yellow homogeneous pigmentation, delicate linear branching, and serpentine vessels. Solitary, red clods are present in some lesions. (D) Dermoscopic finding after 3 months: partially regressed lesions reveal yellow background and linear serpentine vessels. (E) Focally dispersed small dermal granulomas composed of histiocytes and multinuclear giant cells with peripheral arrangement of nuclei (E,F) $(\mathrm{H} \& \mathrm{E}, \times 100)$. (F) Focal emperipolesis is noted in giant cells (arrows) (H\&E, $\times 400$ ). (G) Positive immunohistochemical staining of CD68 in granulomas (magnification $\times 200$ ). Immunohistochemically, granulomas presented with profile CD68+ CD163+/-, and CD1a-.

Although GEH is a benign, self-healing eruption of non-Langerhans cell lineage, follow-up is necessary.

Two age groups of GEH patients are reported: up to 14 years and adults. Brain infiltrations and diabetes insipidus are reported in $3(12.5 \%)$ children up to age 4 years with xanthomatous evolvement. In $5(10.0 \%)$ of the published adult cases, hematological disorders of myeloid lineage such as acute monocytic/monoblastic leukemia ( 2 cases) or chronic myelomonocytic/eosinophilic leukemia ( 3 cases) are reported
(Table 1). In preschool children, diabetes insipidus should be suspected if GEH evolves into xanthoma. In adults, hematological follow-up is suggested.

Clinical differential diagnosis of GEH includes other histiocytic disorders such as Letterer-Siwe disease, juvenile xanthogranuloma (multiple), papular xanthoma, and progressive nodular histiocytosis. Exanthema due to medications and viruses, with separate entity Gianotti-Crosti syndrome and early eruption of guttate psoriasis, are main differentials as well. 
Table 1. Clinical Characteristics of the Published Cases With

Generalized Eruptive Histiocytosis (Histiocytoma) (GEH)

\begin{tabular}{|c|c|c|c|c|c|}
\hline Reference $^{a}$ & $\begin{array}{c}\text { Age at } \\
\text { Onset (yrs) }\end{array}$ & Sex & Location & Course & Associated Finding \\
\hline Wise $(1919)^{c}$ & 22 & M & $\begin{array}{l}\text { Trunk, proximal } \\
\text { part of extremities }\end{array}$ & Lasted 20 years & None \\
\hline $\begin{array}{l}\text { Glauberzon and } \\
\text { Lebedeff }(1952)^{c}\end{array}$ & 34 & $\mathrm{~F}$ & Disseminated & Not defined & None \\
\hline $\begin{array}{l}\text { Calas et al } \\
(1959)^{\mathrm{c}}\end{array}$ & 52 & M & $\begin{array}{l}\text { Face, trunk, } \\
\text { extremities }\end{array}$ & Not defined & None \\
\hline $\begin{array}{l}\text { Baccaredda-Boy } \\
(1960)^{c}\end{array}$ & 33 & M & $\begin{array}{l}\text { Trunk, extremities, } \\
\text { face, mouth }\end{array}$ & No data, died after 19 years & Mutilating polyarthritis \\
\hline Herzberg $(1961)^{c}$ & 30 & $\mathrm{~F}$ & Trunk, extremities & $\begin{array}{l}\text { Spontaneous regression after } \\
13 \text { years }\end{array}$ & Amenorrhea \\
\hline $\begin{array}{l}\text { Winkelmann and } \\
\text { Muller (1963) }\end{array}$ & 51 & $\mathrm{~F}$ & Trunk, extremities & $\begin{array}{l}\text { Number of lesions increased } \\
\text { within } 15 \text { months }\end{array}$ & Osteoarthritis \\
\hline $\begin{array}{l}\text { Winkelmann and } \\
\text { Muller (1963) }\end{array}$ & 58 & M & $\begin{array}{l}\text { Trunk, axillae, } \\
\text { pubic area and } \\
\text { penis, buccal } \\
\text { mucosa }\end{array}$ & $\begin{array}{l}\text { Increasing in number within } \\
4 \text { years }\end{array}$ & None \\
\hline $\begin{array}{l}\text { Winkelmann and } \\
\text { Muller (1963) }\end{array}$ & 38 & $\mathrm{~F}$ & $\begin{array}{l}\text { Face, trunk, } \\
\text { extremities }\end{array}$ & $\begin{array}{l}\text { Cleared gradually after } 12 \\
\text { years }\end{array}$ & None \\
\hline Cramer (1963) & 25 & M & Disseminated & Resistant to steroids & None \\
\hline Pegum (1973) & 42 & M & Trunk, extremities & No regression after 2.5 years & High cholesterol \\
\hline Sohi et al (1979) & 49 & M & Generalized & $\begin{array}{l}\text { Regressed in a few months, } \\
\text { then recurred } 2 \text { years later }\end{array}$ & None \\
\hline $\begin{array}{l}\text { Winkelmann } \\
(1980)\end{array}$ & 3 months & $\mathrm{F}$ & $\begin{array}{l}\text { Extensor limbs, } \\
\text { buttocks }\end{array}$ & $\begin{array}{l}\text { Persisted until at least } 9 \text { years } \\
\text { old }\end{array}$ & Glaucoma and uveitis \\
\hline $\begin{array}{l}\text { Caputo et al } \\
(1981)\end{array}$ & 25 & M & $\begin{array}{l}\text { Thorax, abdomen, } \\
\text { inguinal fold, } \\
\text { proximal } \\
\text { extremities }\end{array}$ & $\begin{array}{l}\text { Resolved spontaneously in } \\
4 \text { years }\end{array}$ & None \\
\hline Aso et al (1982) & 4 & M & Disseminated & $\begin{array}{l}\text { Spontaneous regression of } \\
80 \% \text { lesions after } 2 \text { years }\end{array}$ & None \\
\hline $\begin{array}{l}\text { Arnold et al } \\
(1982)\end{array}$ & 32 & M & Disseminated & $\begin{array}{l}\text { Persisted for } \\
20 \text { years }\end{array}$ & None \\
\hline $\begin{array}{l}\text { Bobin et al } \\
(1983)\end{array}$ & 22 & M & Trunk, extremities & Partial regression within 1 year & None \\
\hline $\begin{array}{l}\text { Idikio and } \\
\text { Hogan (1983) }\end{array}$ & 57 & $\mathrm{~F}$ & $\begin{array}{l}\text { Abdomen, pubic } \\
\text { area, breasts, back, } \\
\text { axillae, face }\end{array}$ & Unchanged in 9 years & Hyperlipidemia type IV \\
\hline $\begin{array}{l}\text { Statham et al } \\
(1984)\end{array}$ & 66 & $\mathrm{~F}$ & $\begin{array}{l}\text { Trunk, extremities, } \\
\text { nasal mucosa }\end{array}$ & $\begin{array}{l}\text { Death } 18 \text { months after the } \\
\text { diagnosis from acute leukemia }\end{array}$ & $\begin{array}{l}\text { Acute leukemia of } \\
\text { monoblastic/histiocytic } \\
\text { origin }\end{array}$ \\
\hline $\begin{array}{l}\text { Caputo et al } \\
(1987)\end{array}$ & 11 months & M & $\begin{array}{l}\text { Trunk, neck, head, } \\
\text { extremities }\end{array}$ & Disappeared in 5 years & None \\
\hline $\begin{array}{l}\text { Caputo et al } \\
(1987)\end{array}$ & 11 months & $\mathrm{F}$ & Face, trunk & Mostly regressed in 6 years & None \\
\hline $\begin{array}{l}\text { Caputo et al } \\
(1987)\end{array}$ & 10 months & M & Trunk, scrotum & Mostly regressed in 2 years & None \\
\hline $\begin{array}{l}\text { Caputo et al } \\
(1987)\end{array}$ & 44 months & $\mathrm{F}$ & Trunk, axillae, face & Mostly regressed in 3 years & None \\
\hline
\end{tabular}

(Table 1 continues) 
Table 1. Clinical Characteristics of the Published Cases With Generalized Eruptive Histiocytosis (Histiocytoma) (GEH) (continued)

\begin{tabular}{|c|c|c|c|c|c|}
\hline Reference $^{a}$ & $\begin{array}{c}\text { Age at } \\
\text { Onset }(y r s)^{b}\end{array}$ & Sex & Location & Course & Associated Finding \\
\hline $\begin{array}{l}\text { Shimizu et al } \\
(1987)\end{array}$ & 24 & M & $\begin{array}{l}\text { Cheeks, later } \\
\text { generalized }\end{array}$ & Regressed in 1 year & None \\
\hline $\begin{array}{l}\text { Sigal-Nahum } \\
\text { et al (1987) }\end{array}$ & 7 & $\mathrm{~F}$ & $\begin{array}{l}\text { Face, buttocks, } \\
\text { extremities }\end{array}$ & $\begin{array}{l}\text { Spontaneous regression and } \\
\text { new crops within few months }\end{array}$ & None \\
\hline $\begin{array}{l}\text { Braun-Falco et al } \\
(1988)\end{array}$ & 19 & M & $\begin{array}{l}\text { Trunk, axillary, } \\
\text { face, throat }\end{array}$ & $\begin{array}{l}\text { Evolved into xanthoma } \\
\text { disseminatum }\end{array}$ & None \\
\hline Grob et al (1988) & 25 & M & Face, axillary & Eruption persisted 2 years & None \\
\hline $\begin{array}{l}\text { Umbert and } \\
\text { Winkelmann } \\
(1989)\end{array}$ & 67 & $\mathrm{~F}$ & Face, trunk, arms & $\begin{array}{l}\text { Slow progression within } 9 \\
\text { years }\end{array}$ & $\begin{array}{l}\text { Hypothyroidism, } \\
\text { normolipemic } \\
\text { xanthelasma, polyclonal } \\
\text { gammopathy }\end{array}$ \\
\hline $\begin{array}{l}\text { Ashworth et al } \\
(1990)\end{array}$ & 21 & M & $\begin{array}{l}\text { Disseminated, } \\
\text { sparing mucosa }\end{array}$ & $\begin{array}{l}8 \text { years continual progression } \\
\text { of the disease }\end{array}$ & $\begin{array}{l}\text { Atopic dermatitis, } \\
\text { asthma }\end{array}$ \\
\hline Saijo et al (1991) & 14 & $\mathrm{~F}$ & Trunk & $\begin{array}{l}\text { Within } 14 \text { months: partial } \\
\text { regression with new lesions }\end{array}$ & None \\
\hline $\begin{array}{l}\text { Stables and } \\
\text { MacKie (1992) }\end{array}$ & 55 & $\mathrm{~F}$ & $\begin{array}{l}\text { Arms, trunk, upper } \\
\text { thighs, face }\end{array}$ & Present for at least 24 months & None \\
\hline Izaki et al (1993) & 1 & M & $\begin{array}{l}\text { Face, neck, upper } \\
\text { arms }\end{array}$ & $\begin{array}{l}\text { Persisted for next } 5 \text { years until } \\
\text { spontaneous resolution }\end{array}$ & None \\
\hline $\begin{array}{l}\text { Goerdt et al } \\
(1994)\end{array}$ & 69 & M & Trunk, extremities & Persisted for at least 5 years & High cholesterol \\
\hline $\begin{array}{l}\text { Repiso et al } \\
(1995)\end{array}$ & 4 & M & $\begin{array}{l}\text { Face, trunk, } \\
\text { proximal } \\
\text { extremities }\end{array}$ & $\begin{array}{l}\text { Evolved into xanthoma } \\
\text { disseminatum }\end{array}$ & $\begin{array}{l}\text { Developed diabetes } \\
\text { insipidus and brain } \\
\text { infiltrations }\end{array}$ \\
\hline $\begin{array}{l}\text { Gibbs and } \\
\text { O'Grady (1996) }\end{array}$ & 41 & M & Face, arms, torso & Lesions persisted for 9 years & Diabetes mellitus type II \\
\hline Jang et al (1999) & 3 months & M & $\begin{array}{l}\text { Face, trunk, groin, } \\
\text { upper and lower } \\
\text { limbs }\end{array}$ & $\begin{array}{l}\text { Spontaneous regression within } \\
2 \text { months; no new lesions } \\
\text { within } 2 \text { years }\end{array}$ & None \\
\hline $\begin{array}{l}\text { Matsushima et al } \\
(1999)\end{array}$ & 5 & $\mathrm{~F}$ & Generalized & $\begin{array}{l}\text { Spontaneous regression within } \\
8 \text { months }\end{array}$ & Rheumatic fever \\
\hline Wee et al (2000) & 9 & M & $\begin{array}{l}\text { Trunk, proximal } \\
\text { extremities }\end{array}$ & $\begin{array}{l}\text { Spontaneous regression of } \\
\text { some lesions with new crops }\end{array}$ & None \\
\hline $\begin{array}{l}\text { Marzano et al } \\
(2002)\end{array}$ & 33 & $\mathrm{~F}$ & Trunk, extremities & Resolved within 6 months & None \\
\hline $\begin{array}{l}\text { Wollenberg et al } \\
(2002)\end{array}$ & 13 & $\mathrm{~F}$ & Abdomen, trunk & Persisted for 3 years & $\begin{array}{l}\text { Coexistence with skin } \\
\text { lesions proven to be } \\
\text { indeterminate cell } \\
\text { histiocytosis }\end{array}$ \\
\hline $\begin{array}{l}\text { Klemke et al } \\
(2003)\end{array}$ & 59 & M & $\begin{array}{l}\text { Trunk, neck, face, } \\
\text { and thighs }\end{array}$ & $\begin{array}{l}\text { Improved under aplasiogenic } \\
\text { regimen }\end{array}$ & $\begin{array}{l}\text { Acute monocytic } \\
\text { leukemia }\end{array}$ \\
\hline $\begin{array}{l}\text { Seward et al } \\
(2004)\end{array}$ & 55 & M & Trunk, extremities & $\begin{array}{l}\text { Several lesions resolved after } \\
\text { cryotherapy }\end{array}$ & None \\
\hline Deng et al (2004) & 39 & M & Face, trunk, limbs & $\begin{array}{l}\text { Spontaneously resolved within } \\
6 \text { months }\end{array}$ & Increased eosinophilia \\
\hline $\begin{array}{l}\text { Mehravaran } \\
(2004)\end{array}$ & 53 & $\mathrm{~F}$ & $\begin{array}{l}\text { Trunk, upper } \\
\text { extremities }\end{array}$ & No follow-up & None \\
\hline $\begin{array}{l}\text { Tamiya et al } \\
(2005)\end{array}$ & 14 months & $\mathrm{F}$ & Trunk, extremities & $\begin{array}{l}\text { Spontaneously resolved within } \\
1 \text { month }\end{array}$ & $\begin{array}{l}\text { Immunoglobulin } \mathrm{G} \text {, } \\
\text { human herpesvirus } 6\end{array}$ \\
\hline
\end{tabular}

(Table 1 continues) 
Table 1. Clinical Characteristics of the Published Cases With Generalized Eruptive Histiocytosis (Histiocytoma) (GEH) (continued)

\begin{tabular}{|c|c|c|c|c|c|}
\hline Reference $^{a}$ & $\begin{array}{c}\text { Age at } \\
\text { Onset (yrs) }\end{array}$ & Sex & Location & Course & Associated Finding \\
\hline $\begin{array}{l}\text { Vázquez-Blanco } \\
\text { et al (2006) }\end{array}$ & 64 & M & $\begin{array}{l}\text { Trunk, extremities, } \\
\text { mucous } \\
\text { membranes }\end{array}$ & $\begin{array}{l}\text { Subsided after } \\
\text { photochemotherapy, but } \\
\text { reappeared }\end{array}$ & None \\
\hline Kiliç et al (2006) & 1 & M & $\begin{array}{l}\text { Face, trunk, } \\
\text { extremities }\end{array}$ & $\begin{array}{l}\text { Stable for } 12 \text { months, partial } \\
\text { regression after } 41 \text { months }\end{array}$ & None \\
\hline $\begin{array}{l}\text { Lan Ma et al } \\
(2007)\end{array}$ & 32 & $\mathrm{~F}$ & $\begin{array}{l}\text { Trunk, extremities, } \\
\text { face }\end{array}$ & $\begin{array}{l}\text { In } 3 \text { months resolved with } \\
\text { PUVA }\end{array}$ & $\begin{array}{l}\text { Eosinophilia in } \\
\text { peripheral blood and in } \\
\text { bone marrow cytology }\end{array}$ \\
\hline Tang et al (2007) & 36 & $\mathrm{~F}$ & $\begin{array}{l}\text { Trunk, abdomen, } \\
\text { extremities }\end{array}$ & $\begin{array}{l}\text { After } 8 \text { years, spontaneous } \\
\text { regression of some lesions } \\
\text { observed }\end{array}$ & None \\
\hline $\begin{array}{l}\text { Fernández-Jorge } \\
\text { et al (2007) }\end{array}$ & 41 & $\mathrm{~F}$ & Trunk and arms & $\begin{array}{l}\text { Spontaneously resolved in } 11 \\
\text { months }\end{array}$ & Hypercholesterolemia \\
\hline $\begin{array}{l}\text { Bajaj and Iqbal } \\
(2008)\end{array}$ & 28 & M & Face, chest, axillae & $\begin{array}{l}\text { Resolved after } 1 \text { week with } \\
\text { liquid nitrogen }\end{array}$ & None \\
\hline $\begin{array}{l}\text { Kwinter and } \\
\text { DeKoven (2009) }\end{array}$ & 53 & $\mathrm{~F}$ & Face, neck, arms & $\begin{array}{l}\text { Resolved after } 8 \text { months with } \\
\text { isotretinoin, then recurred }\end{array}$ & None \\
\hline $\begin{array}{l}\text { Chern et al } \\
(2010)\end{array}$ & 5 months & $\mathrm{F}$ & $\begin{array}{l}\text { Face, trunk, arms, } \\
\text { legs }\end{array}$ & $\begin{array}{l}\text { Spontaneously resolved in } 6 \\
\text { months }\end{array}$ & Mild leukocytosis \\
\hline $\begin{array}{l}\text { Aggarwal et al } \\
(2010)\end{array}$ & 61 & M & Trunk, arms, legs & $\begin{array}{l}\text { Spontaneously resolved with } \\
\text { relapses within } 4 \text { years }\end{array}$ & None \\
\hline $\begin{array}{l}\text { Sharath Kumar } \\
\text { et al (2011) }\end{array}$ & 23 & $\mathrm{~F}$ & $\begin{array}{l}\text { Face, trunk, arms, } \\
\text { legs }\end{array}$ & $\begin{array}{l}\text { Minimal resolution, persisted } \\
5 \text { years }\end{array}$ & None \\
\hline Attia et al (2011) & 48 & $\mathrm{~F}$ & $\begin{array}{l}\text { Upper limbs and } \\
\text { trunk }\end{array}$ & Spontaneously resolved & None \\
\hline $\begin{array}{l}\text { Montero et al } \\
(2012)\end{array}$ & 80 & M & Trunk, abdomen & Resolved after 6 months & $\begin{array}{l}\text { Chronic } \\
\text { myelomonocytic } \\
\text { leukemia }\end{array}$ \\
\hline Verma (2012) & 10 & M & Hands, feet, trunk & $\begin{array}{l}\text { Coexistence with juvenile } \\
\text { xanthogranuloma lesions }\end{array}$ & None \\
\hline $\begin{array}{l}\text { Cardoso et al } \\
(2013)\end{array}$ & 79 & M & Trunk, eyelids & $\begin{array}{l}\text { Spontaneously resolved in } 2 \\
\text { months }\end{array}$ & None \\
\hline $\begin{array}{l}\text { Zamudio Vega } \\
\text { et al (2013) }\end{array}$ & 8 & M & $\begin{array}{l}\text { Face, upper } \\
\text { extremities }\end{array}$ & 8 months unchanged & None \\
\hline Shon et al (2013) & 84 & M & Face, neck, arms & Died after 4 months & $\begin{array}{l}\text { Chronic } \\
\text { myelomonocytic } \\
\text { leukemia }\end{array}$ \\
\hline Kazi et al (2014) & 23 & M & Lower extremities & No follow-up & None \\
\hline $\begin{array}{l}\text { Ghandi et al } \\
(2015)\end{array}$ & 28 & $\mathrm{~F}$ & $\begin{array}{l}\text { Face, trunk, } \\
\text { extremities }\end{array}$ & Spreading within 2 years & None \\
\hline $\begin{array}{l}\text { Ziegler et al } \\
(2015)\end{array}$ & 20 & M & Trunk, extremities & $\begin{array}{l}\text { Complete remission with } \\
\text { imatinib }\end{array}$ & $\begin{array}{l}\text { FIP1L1-PDGFRA- } \\
\text { positive chronic } \\
\text { eosinophilic leukemia }\end{array}$ \\
\hline $\begin{array}{l}\text { Hansel et al } \\
(2015)\end{array}$ & 60 & M & Trunk, extremities & $\begin{array}{l}\text { Remission with PUVA and } \\
\text { topical corticosteroids }\end{array}$ & None \\
\hline $\begin{array}{l}\text { Mahajan et al } \\
(2015)\end{array}$ & 60 & M & Extremities & No follow-up & None \\
\hline Wilk et al (2016) & 64 & $\mathrm{M}$ & Trunk, extremities & Unchanged for several years & None \\
\hline
\end{tabular}

(Table 1 continues) 
Table 1. Clinical Characteristics of the Published Cases With Generalized Eruptive Histiocytosis (Histiocytoma) (GEH) (continued)

\begin{tabular}{|c|c|c|c|c|c|}
\hline Reference $^{a}$ & $\begin{array}{c}\text { Age at } \\
\text { Onset (yrs) }\end{array}$ & Sex & Location & Course & Associated Finding \\
\hline Piney et al (2016) & 28 & M & $\begin{array}{l}\text { Face, trunk, } \\
\text { extremities }\end{array}$ & $\begin{array}{l}\text { Resistant to imatinib, } \\
\text { interferon alpha, anakinra; } \\
\text { resolved after PUVA therapy }\end{array}$ & Arthralgia \\
\hline $\begin{array}{l}\text { Alperovich et al } \\
(2017)\end{array}$ & 3 & $\mathrm{~F}$ & Trunk, face & $\begin{array}{l}\text { 6-month follow-up: CNS } \\
\text { lesions-xanthomata }\end{array}$ & Diabetes insipidus \\
\hline Arif et al (2017) & 26 & $\mathrm{~F}$ & Face, trunk, arms & $\begin{array}{l}\text { Persisted and coalesced within } \\
3 \text { months }\end{array}$ & None \\
\hline Kar et al (2018) & 6 & $\mathrm{~F}$ & Face, axillae, trunk & Spontaneously resolved & None \\
\hline $\begin{array}{l}\text { Kaçar et al } \\
(2018)\end{array}$ & 19 months & $\mathrm{F}$ & $\begin{array}{l}\text { Diaper area, } \\
\text { extremities, trunk }\end{array}$ & Spontaneously resolved & None \\
\hline $\begin{array}{l}\text { Costin et al } \\
(2019)\end{array}$ & 24 & $\mathrm{~F}$ & $\begin{array}{l}\text { Trunk, neck and } \\
\text { proximal upper } \\
\text { extremities }\end{array}$ & Unchanged after 1 year & None \\
\hline $\begin{array}{l}\text { Takahashi et al } \\
(2019)\end{array}$ & 1 & M & Trunk & After 1 year partially regressed & None \\
\hline $\begin{array}{l}\text { Kobayashi et al } \\
(2019)\end{array}$ & 7 months & M & $\begin{array}{l}\text { Neck, trunk, } \\
\text { extremities }\end{array}$ & $\begin{array}{l}\text { 2-year follow-up; lesion } \\
\text { evolved into xanthoma } \\
\text { disseminatum; resistance to } \\
\text { chemotherapy }\end{array}$ & Diabetes insipidus \\
\hline
\end{tabular}

${ }^{a}$ Full references are provided in the supplementary content, which is appended to the pdf.

${ }^{\mathrm{b}}$ Age is given in years except where indicated as months.

In 1963 Winkelmann and Muller reported 3 cases of GEH, but some cases with cutaneous and histological abnormalities consistent with GEH were reported before their report, under different names (Sohi et al, Dermatologica. 1979;159(6):71-75; Bobin et al, Ann Dermatol Venereol. 1983;110(10):817-824).

A case describing dermoscopy of GEH lesions resembling molluscum contagiosum in an infant has been published [1]. A homogeneous orange-yellow pattern with an erythematous border described as "setting-sun" was recognized. Histology revealed histiocytic cells with foamy xanthomatous cytoplasm [1]. The dermoscopic finding in our case presented with orange-yellow homogeneous pigmentation, delicate linear branching, serpentine vessels, and solitary, red clods. Histology revealed histiocytic cells forming granulomas.

The dermoscopic differential diagnosis of GEH is broad and encompasses juvenile xanthogranuloma, cutaneous sarcoidosis, necrobiosis lipoidica, granuloma annulare (palisading granuloma histological subtype), elastosis perforans serpiginosa, granulomatous rosacea, annular elastolytic giant cell granuloma, and rheumatoid nodules. Among infective diseases, lupus vulgaris, cutaneous leishmaniasis, borderline tuberculoid leprosy, and Majocchi granuloma are the most important differentials [2]. Combining clinical, dermoscopic, and histological findings is of greatest importance in any of the diseases mentioned.

\section{Conclusions}

Our case emphasizes the importance of dermoscopic examination in the everyday practice of dermatologists. Further studies of skin histiocytic disorders are required in order to establish all dermoscopic criteria.

\section{Acknowledgment}

The authors thank Mrs. Ana Ivkovic from the Institute of Oncology and Radiology of Serbia, Belgrade, Serbia, and Mr. William Russell-Edu from the European Institute of Oncology, Milan, Italy, for providing scientific literature.

\section{References}

1. Kaçar N, Demirkan N, Duygulu Ş. Generalized eruptive histiocytosis diagnosed in light of dermoscopic findings. Int J Dermatol. 2018;57(3):355-357. https://doi.org/10.1111/ijd.13867

2. Erricheti E, Stinco E. Dermoscopy in general dermatology: a practical overview. Dermatol Ther (Heidelb). 2016;6(4):471-507. https:// doi.org/10.1007/s13555-016-0141-6 


\section{Supplementary Material: Table 1 References}

1. Wise F. Multiple endothelioma of the skin. Am J Med Sci. 1919;157:236-253.

2. Glauberzon SA, Lebedeff YA. Multiple plane dermato-fibrome (fibroma simplex, derma-fibroma lenticulare). Vesternick Venerologie Dermatologie. 1952;35. Excerpta Med (Section 13)6:68.

3. Calas E, Tramier G, Coulier L. Reticulose benigne (histiocytomes multiples dissemines). Bull Soc Fr Derm Syph. 1959;66:120-121.

4. Baccaredda-Boy A. Paraxathomatose (thesaurotische) system Histiocytose. Hautarzt. 1960;11:58-63.

5. Herzberg JJ. Eruptive symmetrisch angeordnete eosinophile Granulome der Haut. Arch Klin Exp Dermatol. 1961;212:282-297.

6. Winkelmann RK, Muller SA. Generalized eruptive histiocytoma: a benign papular histiocytic reticulosis. Arch Dermatol. 1963;88:586-596. https://doi.org/10.1001/archderm.1963. 01590230094014

7. Cramer HJ. Cramer HJ. Multiple Reticulo-histiocytome der Haut ohne nachweisbare Zweiterkrankung. Hautarzt. 1963;14:297302.

8. Pegum JS. Generalized eruptive histiocytoma. Proc R Soc Med. 1973;66:59-60.

9. Sohi AS, Tiwari VD, Subramanian CS, Chakraborty M. Generalized eruptive histiocytoma: a case report with a review of the literature. Dermatologica. 1979;159(6):71-75.

10. Winkelmann RK, Kossard S, Fraga S. Eruptive histiocytoma of childhood. Arch Dermatol. 1980;116(5):565-570.

11. Caputo R, Alessi E, Allegra F. Generalized eruptive histiocytoma: a clinical, histologic, and ultrastructural study. Arch Dermatol. 1981;117(4):216-221. https://doi.org/10.1001/archderm. 1981.01650040032016

12. Aso K, Kondo Y, Watanabe S. A case of pediatric generalized eruptive histiocytoma [in Japanese]. Nihon Hifuka Gakkai Zasshi. 1982;92(2):115-120.

13. Arnold ML, Wirth H, Anton-Lamprecht I, Petzoldt D. Verallgemeinert Eruptive Histiozytome. Hautarzt. 1982;33(8):428-437.

14. Bobin P, Carsuzaa F, Seurat P, Lucas D. Histiocytome eruptif generalise. A propos d'un cas. Revue de la literature. Ann Dermatol Venereol. 1983;110(10):817-824.

15. Idikio H, Hogan J. Generalized eruptive histiocytoma: report of a case and electron microscopic finding of Michaelis-Gutmann bodies. Clin Exp Dermatol. 1983;8(6):625-630. https://doi. org/10.1111/j.1365-2230.1983.tb01832.x

16. Statham BN, Fairris GM, Cotterill JA. Atypical eruptive histiocytosis-a marker of underlying malignancy? Br J Dermatol. 1984;110 (1):103-105. https://doi.org/10.1111/j.1365-2133.1984.tb07319.x.

17. Caputo R, Ermacora E, Gelmetti C, Berti E, Giani E, Nigro A. Generalized eruptive histiocytoma in children. J Am Acad Dermatol. 1987;17(3 ):449-454. https://doi.org/10.1016/s01909622(87)70228-x

18. Shimizu N, Ito M, Sato Y. Generalized eruptive histiocytoma: an ultrastructural study. J Cutan Pathol. 1987:14(2):100-105. https:// doi.org/10.1111/j.1600-0560.1987.tb00480.x

19. Sigal-Nahum M, Gaulier A, Basset F, Carado Y, Sigal S. Histiocytose non $\mathrm{X}$ (histiocytome eruptif generalise?) marquee par l'OKT6, a filaments de vimentine. A propos d'un cas. Ann Dermatol Venereol. 1987;114(2):211-221.

20. Braun-Falco O, Korting HC, Zienicke H, Klovekorn W. Eruptive Histiozytome und Xanthoma disseminatum als Manifestationsformen derselben Erkrankung? Hautarzt. 1988;39(10):652-657.
21. Grob JJ, Collet AM, Horchowski N, Andrac L, Chouquet G, Bonerandi JJ. Histiocytome eruptif generalise de Winkelmann et Muller. Etude ultrastructurale et immunohistochimique. Ann Dermatol Venereol. 1988;115(11):1202-1204.

22. Umbert I, Winkelmann RK. Eruptive histiocytoma. J Am Acad Dermatol. 1989;20(5 Pt 2):958-964. https://doi.org/10.1016/ s0190-9622(89)70119-5

23. Ashworth J, Archard L, Woodrow D, Cream JJ. Multiple eruptive histiocytoma cutis in an atopic. Clin Exp Dermatol. 1990;15(6):454-456. https://doi.org/10.1111/j.1365-2230.1990. tb02143.x

24. Saijo S, Hara M, Kuramoto Y, Tagami H. Generalized eruptive histiocytoma: a report of a variant case showing the presence of dermal indeterminate cells. J Cutan Pathol. 1991;18(2):134-136. https://doi.org/10.1111/j.1600-0560.1991.tb00141.x

25. Stables GI, MacKie R. Generalized eruptive histiocytoma. $\mathrm{Br} J$ Dermatol. 1992;126(2):196-199. https://doi.org/10.1111/j.13652133.1992.tb07821.x

26. Izaki S, Kitamura K, Arai E. Generalized eruptive histiocytoma: report of a pediatric case. J Dermatol. 1993;20:105-108. https:// doi.org/10.1111/j.1346-8138.1993.tb03840.x

27. Goerdt S, Bonsmann G, Sunderkötter C, Grabbe S, Luger T, Kolde G. A unique non-Langerhans cell histiocytosis with some features of generalized eruptive histiocytoma. J Am Acad Dermatol. 1994;31(2 Pt2):322-326. https://doi.org/10.1016/s01909622(94)70164-4

28. Repiso T, Roca-Mirales M, Kanitakis J, Castells-Rodellas A. Generalized eruptive histiocytoma evolving into xanthogranuloma disseminatum in a 4-year-old boy. Br J Dermatol. 1995;132(6):978982. https://doi.org/10.1111/j.1365-2133.1995.tb16959.x

29. Gibbs N, O'Grady T. Progressive eruptive histiocytoma. J Am Acad Dermatol. 1996;35(2 Pt2):323-325. https://doi.org/10.1016/ s0190-9622(96)90660-x

30. Jang KA, Lee HJ, Choi JH, Sung KJ, Koh JK, Moon KC. Generalized eruptive histiocytoma of childhood. Br J Dermatol. 1999;140(1):168-192. https://doi.org/10.1046/j.13652133.1999.02634.x

31. Matsushima Y, Ohnishi K, Ishikawa O. Generalized eruptive histiocytoma of childhood associated with rheumatic fever. Eur J Dermatol. 1999;9(7):548-550.

32. Wee SH, Kim HS, Chang SN, Kim DK, Park WH. Generalized eruptive histiocytoma: a pediatric case. Pediatr Dermatol. 2000;17(6):453-455. https://doi.org/10.1046/j.15251470.2000.01842.x

33. Marzano AV, Facceti M, Caputo R. Guess what! Diagnosis: generalized eruptive histiocytosis (histiocytoma). Eur J Dermatol. 2002;12(2);205-206.

34. Wollenberg A, Burgdorf WH, Schaller M, Sander C. Long-lasting "Christmas tree rash" in an adolescent: isotopic response of indeterminate cell histiocytosis in pityriasis rosea? Acta Derm Venereol. 2002;82(4):288-291. https://doi. org/10.1080/000155502320323270

35. Klemke CD, Dippel E, Geilen CC, et al. Atypical generalized eruptive histiocytosis associated with acute monocytic leukemia. J Am Acad Dermatol. 2003;49(5 Suppl ):S233-S236. https://doi. org/10.1016/s0190-9622(03)00037-9

36. Seward J, Malone J, Callen J. Generalized eruptive histiocytosis. J Am Acad Dermatol. 2004;50(1):116-120. https://doi. org/10.1016/s0190-9622(03)02789-0 
37. Deng YJ, Hao F, Zhou CL, et al. Generalized eruptive histiocytosis: a possible therapeutic cure? Br J Dermatol. 2004;150(1):171173. https://doi.org/10.1111/j.1365-2133.2004.05699.x

38. Mehravan M. Quiz: July 2004. Generalized eruptive histiocytosis (GEH). Available from: http://www.iranderma.com/quiz-jul04. htm. Accessed November 23, 2019.

39. Tamiya H, Tsuruta D, Takeda E, Moriwaki K, Kobayash H, Ishii $\mathrm{M}$. Generalized eruptive histiocytoma with rapid progression and resolution following exanthema subitum. Clin Exp Dermatol. 2005;30(3):294-307. https://doi.org/10.1111/j.13652230.2005.01738.x

40. Vázquez-Blanco M, Peteiro C, Toribio J. Histiocitoma eruptivo generalizado. Actas Dermosifiliogr. 2006;97(1):35-38. https://doi. org/10.1016 / S0001-7310 (06) 73345-2

41. Kiliç A, Kulcu Cakmak S, Gonul M, Gul U, Demirel O, Demiriz M. Generalized eruptive histiocytoma: a pediatric case. Eur J Dermatol. 2006;16(6):694-695.

42. Lan Ma H, Metze D, Luger TA, Steinhoff M. Successful treatment of generalized eruptive histiocytoma with PUVA. J Dtsch Dermatol Ges. 2007;5(2):131-134. https://doi.org/10.1111/j.16100387.2007.06178.x

43. Tang X, Shen H, Xu A, et al. Spontaneous regression of generalized eruptive histiocytosis: possible involvement of apoptosis? Int J Dermatol. 2007;46(10):1073-1075. https://doi.org/10.1111/ j.1365-4632.2006.03045.x

44. Fernández-Jorge B, Goday-Buján J, Del Pozo Losada J, Alvarez-Rodríguez R, Fonseca E. A case of generalized eruptive histiocytosis. Acta Derm Venereol. 2007;87(6):533-536. https://doi. org/10.2340/00015555-0212

45. Bajaj DR, Iqbal MP. Generalized eruptive histiocytosis presenting with warty lesions on face. J Coll Physicians Surg Pak. 2008;18(2):110-112. https://doi.org/02.2008/JCPSP.110112

46. Kwinter J, DeKoven J. Generalized eruptive histiocytoma treated with isotretinoin. J Cutan Med Surg. 2009;13(3):146-150. https:// doi.org/10.2310/7750.2008.07091

47. Chern E, Yau D, Ho JC. Generalized eruptive histiocytosis in an infant. Acta Derm Venereol. 2010;90(2):204-205. https://doi.org/ 10.2340/00015555-0805

48. Aggarwal K, Gupta S, Jain VK, Sen R, Gupta S. Generalized eruptive histiocytoma. Indian Dermatol Online J. 2010;1(1):27-29. https://doi.org/10.4103/2229-5178.73255

49. Sharath Kumar BC, Nandini AS, Niveditha SR, Gopal MG; Reeti. Generalized eruptive histiocytosis mimicking leprosy. Indian J Dermatol Venereol Leprol. 2011;77(4):498-502. https://doi. org/10.4103/0378-6323.82413

50. Attia A, Seleit I, El Badwy N, Bakry O, Yassien H. Generalized eruptive histiocytoma. J Dermatol Case Rep. 2011;5(3):53-55. https://doi.org/10.3315/jdcr.2011.1076.

51. Montero I, Gutiérrez-González E, Ginarte M, Toribio J. Histiocitosis eruptiva generalizada en paciente con leucemia mielomonocítica crónica. Actas Dermo-Sifiliográficas. 2012;103(7):643-644. https://doi.org/10.1016/j.ad.2012.01.013

52. Verma S. Generalized eruptive histiocytomas and juvenile eruptive xanthogranulomas in a 10 -year-old boy: a potpourri of exotic terms indicating the need for unification. Int J Dermatol. 2012;51(4):445-447. https://doi.org/ 10.1111/j.13654632.2011.05288.x

53. Cardoso F, Serafini NB, Reis BD, Nuñez MD, Nery JA, Lupi O. Generalized eruptive histiocytoma: a rare disease in an elderly patient. An Bras Dermatol. 2013;88(1):105-108. https://doi. org/10.1590/s0365-05962013000100015
54. Zamudio Vega P, Ysita Morales A, Medaro Mora Huerta J. Un caso de histiocytoma eruptivo generalizado. Revista Mex Ped. 2013;80(5):191-194. Available from: http://www.medigraphic. com/rmp.

55. Shon W, Peters MS, Reed KB, Ketterling RP, Dogan A, Gibson LE. Atypical generalized eruptive histiocytosis clonally related to chronic myelomonocytic leukemia with loss of Y chromosome. J Cutan Pathol. 2013; 40(8):725-729. https://doi.org/10.1111/ cup. 12168 .

56. Kazi N, Bernert R, Moussa C, Magro C. A case of generalized eruptive histiocytosis in a 23-year-old man. Dermatol Online J. 2014;20(8):13030/qt39v75449.

57. Ghandi N, Daklan S, Goodarzi A, Kamyab Hesari K, Ghanadan A. Generalized eruptive histiocytosis: a case report. Iran J Dermatol. 2015;18(1):33-35. Available from: http://iranjd.ir/article_ 98250.html

58. Ziegler B, Peitsch WK, Reiter A, Marx A, Goerdt S, Géraud C. Generalized eruptive histiocytosis associated with FIP1L1-PDG FRA-positive chronic eosinophilic leukemia. JAMA Dermatol. 2015;151(7):766-769. https://doi.org/10.1001/jamadermatol. 2015.0154

59. Hansel G, Schönlebe J, Tchernev G, Chokoeva AA, Lotti T, Wollina U. Generalized eruptive histiocytoma in adult patient. J Biol Regul Homeost Agents. 2015;29(1 Suppl):15-17.

60. Mahajan RS, Shah AC, Pasle RK, Bilimoria FE. Asymptomatic papular eruption in a 60 year old man. Indian J Dermatol. 2015;60(5):516-517. https://doi.org/10.4103/0019-5154.164391

61. Wilk M, Zelger BG, Zelger B. Generalized eruptive histiocytosis with features of multinucleate cell angiohistiocytoma. Am J Dermatopathol. 2016;38(6):470-472. https://doi.org/10.1097/ DAD.0000000000000402

62. Pinney SS, Jahan-Tigh RR, Chon S. Generalized eruptive histiocytosis associated with a novel fusion in LMNA-NTRK1. Dermatol Online J. 2016;22(8):13030/qt07d3f2xk.

63. Alperovich R, Grassino PT, Asial R, Pasteris L, Boente MD. Histiocitosis eruptiva generalizada-xantogranuloma juvenil: espectro clínico en un paciente pediátrico. Arch Argent Pediatr. 2017;115(2):e116-e119. https://doi.org/10.5546/aap.2017.e116

64. Arif T, Adil M, Amin SS, Dorjay K. Generalized eruptive histiocytosis simulating leprosy: a case report with current status of classification of histiocytoses and antigenic markers. Indian J Paediatr Dermatol. 2017;20(20):1-4. https://doi.org/10.4103/ ijpd.IJPD_124_16

65. Kar C, Biswas R, Benerjee S, Sarkar P, Shome K, Das KD. Generalized eruptive histiocytosis in a child: a rare entity. Indian J Dermatopathol Diagn Dermatol. 2018;5(2):143-145. https://doi. org/10.4103/ijdpdd.ijdpdd_1_18

66. Kaçar N, Demirkan N, Duygulu Ș. Generalized eruptive histiocytosis diagnosed in the light of dermoscopic findings. Int J Dermatol. 2018;57(3):355-357. https://doi.org/10.1111/ijd.13867

67. Costin A, Cerejeira D, Alves J. Generalised eruptive histiocytosis: case report. Australas J Dermatol. 2019;60(4):e314-e316. https:// doi.org/10.1111/ajd.13062

68. Takahashi S, Muto J, Takama H, et al. Generalized eruptive histiocytoma: pediatric case report and review of the published work. $J$ Dermatol. 2019;46(11):e407-e408. https://doi.org/10.1111/13468138.15005

69. Kobayashi K, Kurihara Y, Yamagami J, et al. Generalized eruptive histiocytoma developing into xanthoma disseminatum with central diabetes insipidus. J Dermatol. 2019;46(8):e281-e283. https:// doi.org/10.1111/1346-8138.14845 